\title{
Damping Behavior of 3D Woven Metallic Lattice Materials
}

\author{
Stephen M. Ryan ${ }^{\mathrm{a}}$, Stefan Szyniszewski ${ }^{\mathrm{b}}$, Seunghyun $\mathrm{Ha}^{\mathrm{c}, \mathrm{d}}$, Rui Xiao ${ }^{\mathrm{f}}$, Thao D. Nguyen ${ }^{\mathrm{f}}$, Keith \\ W. Sharp ${ }^{\mathrm{e}}$, Timothy P. Weihs ${ }^{\mathrm{a}}$, James K. Guest ${ }^{\mathrm{c}}$, and Kevin J. Hemker, ${ }^{\mathrm{a}, \mathrm{f}, 1}$ \\ ${ }^{a}$ Dept. of Materials Science and Engineering, Johns Hopkins University, Baltimore, USA \\ ${ }^{\mathrm{b}}$ Dept. of Civil Engineering, University of Surrey, Guildford, UK \\ ${ }^{c}$ Dept. of Civil Engineering, Johns Hopkins University, Baltimore, USA \\ ${ }^{\mathrm{d}}$ Dept. of Ocean Engineering, Korea Maritime and Ocean University \\ ${ }^{\mathrm{e}}$ Saertex USA, LLC, Huntersville, USA \\ ${ }^{\mathrm{f}}$ Dept. of Mechanical Engineering, Johns Hopkins University, Baltimore, USA
}

\begin{abstract}
$\mathrm{Cu}$ and $\mathrm{NiCr}$ metallic lattice materials of two different micro-architectures were manufactured with a 3D weaving process. Dynamic mechanical analysis experiments demonstrated that the damping properties of these materials are much greater than their bulk counterparts and were found to have damping loss coefficients comparable to polymers, but with much higher maximum use temperatures. The magnitude of the damping phenomenon is characterized experimentally, and the importance of Coulomb (frictional) damping and inertial damping are investigated using a finite element model.
\end{abstract}

Keywords: damping, metallic lattice material, 3-D weaving, micro-architected materials, design

\footnotetext{
${ }^{1}$ Corresponding author: 101 Latrobe Hall, Johns Hopkins University, Baltimore, MD 21218, USA. hemker@jhu.edu
} 
Research on metal foams has demonstrated the potential of porous metals to enhance mechanical characteristics, such as energy dissipation [1-4], buckling mitigation [5,6], and bending rigidity (especially for sandwich panels [7]). The random pore structure of metallic foams [8] is contrasted with micro-architected materials, which have been introduced as highly efficient materials with increased promise in multifunctional applications due to their controlled pore structure [9-15]. Three-dimensional (3D) weaving of metallic wires provides an efficient and cost effective means of creating meaningful volumes of metallic micro-architected "lattice materials". The pore structure may be tailored by designing the wire architecture to optimize specific properties, e.g. fluid permeability [16], and wires may be bonded to create stiff microlattices [17]. The current study examines the damping properties of these 3D woven metallic lattice materials.

Vibrations that occur within high speed rotary devices, such as turbines, can lead to excessive wear and fatigue [18], and measures to reduce vibrations within these components hold significant interest. Dissipation of vibrational kinetic energy under dynamic loading [19-21] is essential for the attenuation of unwanted vibrations and oscillations that can lead to premature failure. Although polymeric materials typically offer excellent damping properties, they are not feasible in high temperature environments and there is a need for non-polymeric materials that can dampen vibrations at high operating temperatures without the use of a damping fluid.

Randomly oriented, metallic wire mesh dampers were initially developed for use in the space shuttle main engine high-pressure fuel turbo pump [22]. The rotodynamic instability of the rocket engine turbopump, characterized by large and damaging subsynchronous whirling motions, was mitigated with a wire mesh mechanical damper that offered improved stability by reducing the reaction forces on the supports [22]. More recently, General Electric (GE) 
developed oil-free dampers for applications in turbomachinery [23,24]. A knitted mesh of interlocking loops of copper wires was compressed into a toroidal shape with $25 \%$ mesh density and employed as a bearing support damper that depended on the excitation frequency.

The current research focuses on micro-architected metallic lattice materials manufactured through a non-crimp 3D weaving technique $[25,26]$. The lattice materials are made from $202 \mu \mathrm{m}$ diameter wires of either $\mathrm{OFHC} \mathrm{Cu}$ (oxygen free high conductivity copper) or Chromel-A (a $\mathrm{NiCr}$ alloy of $80 \%$ nickel and 20\% chromium) (Figure 1). The 3D weaving process essentially stacks pairs of orthogonally oriented warp and fill wires, which are then bound in process, with Z-wires that pass through the thickness (Figure 1 and 2). Two different architectures were considered. The first architecture had wires were placed in all candidate locations within the fiber architecture and is referred to as the 'standard' weave. The second, lower density architecture selectively left some locations with the fiber architecture unfilled, and is referred to as a 'modified' weave. Topology optimization for fluid permeability and in plane shear stiffness informed the choice of which positions to leave unfilled. Specifics about these architectures and the topology optimization approach can be found in [16,27] and images of the 'standard' and 'modified' weaves are shown in figures 1 and 3, respectively. The damping properties of these materials were measured over a range of frequencies, from $1 \mathrm{~Hz}$ to $200 \mathrm{~Hz}$.

The ratio of the loss modulus $k_{s}{ }^{\prime \prime}$ to the storage modulus $k_{s}{ }^{\prime}$ was employed for damping quantification. This ratio is equivalent to the tangent of the phase lag, $\phi$, between the force and displacement oscillations in the idealized spring and dashpot system (see p.60 in [28]), and it is referred to as the loss coefficient: 


$$
\eta=\tan \phi=\frac{k_{s}^{\prime \prime}}{k_{s}^{\prime}}
$$

Loss and storage modulus were measured using a TA Instruments Q800 Dynamic Mechanical Analyzer (DMA) and were calculated from LS-DYNA simulation results based on the theory in [28] as:

$$
\begin{aligned}
& k_{s}^{\prime}=\frac{P_{a}^{\prime}}{X_{a}} \\
& k_{s}^{\prime \prime}=\frac{P_{a}^{\prime \prime}}{X_{a}}
\end{aligned}
$$

where $X_{a}$ is the displacement amplitude (maximum displacement), $P_{a}{ }^{\prime}$ is the force at the peak displacement (in-phase reaction), and $P_{a}{ }^{\prime \prime}$ is the force at the zero displacement (out-of-phase reaction) [28]. The loss coefficient can be approximated to other damping measures for single degree of freedom systems as follows [28-31]:

$$
\eta=\frac{\Delta U}{2 \pi U_{\max }}=2 \zeta \sqrt{1-\zeta^{2}}
$$

where $\Delta U$ is the dissipated energy per cycle, $U_{\max }$ is the elastic energy at peak force, and $\zeta$ is the critical damping ratio. The loss coefficient, $\eta$, was experimentally measured with the DMA in order to characterize the overall damping properties of the material [32]. Computational simulations were then employed to investigate the damping mechanisms, specifically Coulomb (frictional) damping and inertial damping, in order to estimate their relative contributions to the loss coefficient. The frictional energy dissipation mechanism is attributed to wire on wire sliding. The inertial damping mechanism is envisaged to result from out of phase wire movements in the weave, and includes energy dissipation through collision of wires. 
Samples for these dynamic tests were prepared by wire electrical discharge machining (EDM) from the bulk 3D weaves in order to minimize damage caused by the cutting process. Samples were cut to widths of 10 and $15 \mathrm{~mm}$ and a minimum length of $25 \mathrm{~mm}$. The unsupported length was $17.5 \mathrm{~mm}$, and the remainder of the material was clamped at each end in the DMA (Figure 1). The grips were tightened with one bolt per grip by applying $0.3 \mathrm{~N}-\mathrm{m}$ torque. One of the clamps was then subjected to a sinusoidal oscillation with amplitude of $20 \mu m$ and with frequency varied from 1 to $200 \mathrm{~Hz}$. The amplitude was chosen to keep the internal wire stresses below their yield stress.

Ten different woven samples were tested over a range of 1 to $200 \mathrm{~Hz}$. Each sample was tested in four different orientations by flipping and rotating the samples between tests to ensure repeatability, resulting in greater than 40 measurements. No systematic frequency dependence of the loss coefficient was observed as illustrated in the insert in Fig. 2, and the measurements were averaged for each frequency. The compilation of experimental data presented in Fig. 2 indicates that the measured damping loss coefficients of our woven lattices are an order of magnitude greater than for a solid sample of the same material. The loss coefficients ranged from 0.24 to 0.26 for the $\mathrm{Cu}$ lattices and from 0.18 to 0.19 for the $\mathrm{NiCr}$ lattices, which were all significantly greater than the value of $0.01 \pm 0.03$ that was measured for solid $\mathrm{Cu}$ and literature values of 0.001 for solid $\mathrm{NiCr}$ [33]. The standard deviation of the measurements of the woven materials ranged from 0.07 to 0.15 . We believe that this is attributed to the fact that it is difficult to clamp each sample in the exact same position between each frequency sweep.

Dynamic tests of NiCr-modified architecture weaves were simulated using LS-DYNA software [34] in order to elucidate the energy dissipation mechanisms. A model with the dimensions of $10 \times 17.5 \mathrm{~mm}$ (matching the size of the DMA experimental samples) was 
generated with all wires explicitly included (Figure 3). Optical characterization of the manufactured samples revealed gaps between the wires (e.g., Figure 1c) and therefore multiple permutations of the wire positions were considered. These included: (i) a tightly-packed, 'perfect' model where all wires are tangent and there are no unintended gaps between wire junctions, referred to as the zero-gap model; and (ii) a model featuring gaps in between all wires where the vertical gaps were varied (Figure 3). Although the distribution of gaps in the manufactured sample is stochastic, as discussed in [17] and seen in Figure 1c, a simplification is made herein to assume uniform gap sizes in each direction that are based on optical measurements of real samples. The mean spacing values from the optical measurements that were employed in the model were $99 \mu m$ in the warp direction, $10 \mu m$ in fill direction, and $47 \mu m$ vertical (z-direction) gaps between warp and fill wire pairs, shown to scale in Figure 3 for the modified weave architecture. While neither of these models is exactly representative of a manufactured sample, since all simulated wires are perfectly straight and free of residual stresses, the models provide insight into the damping mechanisms at play in the $3 \mathrm{D}$ woven materials.

The nodes on one end were fully clamped in order to achieve a fixed boundary and an oscillatory force load was imposed on the opposite end with a virtually massless vertical elastic shell. The mass of the loading plate was less than $0.1 \%$ of the specimen's mass in order to ensure that its inertia did not affect the simulation results. A sinusoidal load at $70 \mathrm{~Hz}$ was applied to the top of the loading plate, such that oscillatory displacements of $20 \mu m$ were achieved. An explicit solver was used for all simulations, with contact and friction between wires captured through the use of the general contact algorithm (CONTACT AUTOMATIC GENERAL [34]). A very small time step of 8.4 ns was used to track contact points, and beam elements representing the wires 
were fully integrated. A static coefficient of friction $f_{s}=0.44$ [35] and dynamic coefficient of friction $f_{d}=0.2$ [36] was employed in the simulations. The ratio of dynamic to static coefficient of friction is consistent with the ratios given for other metallic materials [37]. To match observations from dynamic friction tests for other metallic materials such as mild steel [37], the transition from static to dynamic coefficient of friction was modeled using an exponential decay function that decays to the dynamic coefficient of friction for contact sliding velocities larger than $25 \mathrm{~mm} / \mathrm{s}$. The elastic modulus of $\mathrm{NiCr}$ was estimated to be $E=130 \mathrm{GPa}$ from initial elastic loading during tensile tests of a single $\mathrm{NiCr}$ wire. Results of the dynamic analysis were then used with equations (1)-(3) to estimate the loss coefficient. Internal damping of the $\mathrm{NiCr}$ material was not considered in the model as it is negligible compared to the observed loss coefficients in the materials.

Simulations of the NiCr modified architecture lattice predicted absolute loss coefficients with magnitudes that were the right order of magnitude, though only about half of the experimental values. The fact that the simulations captured the more than $10 \mathrm{X}$ increase over bulk samples is encouraging and the observation that the simulated losses are systematically lower than the measured values points to the influence of stochastic irregularities in the underlying architecture on the dynamic response. The computational model (Figure 3) employed idealized geometries of the wires (all warp and fill wires were straight, and Z-wires had $90^{\circ}$ bends), while Figure 1 shows that wires were not perfectly straight and were often in contact with other wires, despite the gaps between wires. Similarly, Zhao et al. [16] and Zhang et al. [17] have recently demonstrated that manufacturing irregularities and the stochastic nature of wire geometries need to be considered to accurately model the permeability and stiffness of these lattice materials. The models with idealized geometries were nevertheless useful for a 
preliminary study of the sensitivity of the damping loss coefficient on two key parameters: coefficient of friction and average gap size.

The effect of the coefficient of friction on damping was found to be dependent on the assumed gap sizes. As is illustrated in Table 1, doubling the coefficient of friction increased the simulated loss coefficient by one third for both the zero-gap model, and 10\% when gaps were only incorporated in the warp and fill directions. By contrast, simulations that included gaps in all three directions showed no appreciable change in the simulated loss coefficient when the friction coefficient was doubled. Intuitively this result indicates that friction-based damping is activated in tightly packed lattices, while inertial-based damping is always present but likewise dependent on gap sizes. It is worth noting that the coefficient of friction for $\mathrm{Cu}\left(f_{S} \mathrm{Cu}=1.1\right.$ [37]) is larger than that of $\mathrm{NiCr}\left(f_{S} \mathrm{NiCr}=0.44\right.$ [35] $)$ and that the measured loss coefficients for the $\mathrm{Cu}$ lattices were consistently and significantly higher than for the $\mathrm{NiCr}$ lattices. This suggests that even with inherent manufacturing irregularities, damping of woven lattice materials is influenced by friction.

Changes in the mean gaps sizes were further investigated and found to have a negligible effect on the simulated loss coefficient. A model using mean gap sizes similar to those present in the experimental samples exhibited a simulated loss coefficient of 0.06 . Doubling the z-direction gaps (from 47 to 94 microns) decreased the simulated loss coefficient from 0.06 to 0.05 . Halving the z-direction gaps (from 47 to 23.5 microns) also decreased the simulated loss coefficient from 0.06 to 0.05 . The variations between simulated loss coefficients indicated that within the considered loading frequency range the inertial damping is not particularly sensitive to uniformly distributed (non-stochastic) gaps sizes, as long as there is sufficient space for the wire movement to occur. 
The experimental results (Figure 2) clearly demonstrate that 3D woven metallic lattice materials hold promise as damping materials. The majority of conventional damping materials with comparable loss coefficients, such as polymers, are restricted to significantly lower temperatures, whereas the $\mathrm{NiCr}$ wires have a maximum service temperature of $1175^{\circ} \mathrm{C}$ [38]. A property correlation plot of the damping properties measured in this work with the maximum service temperatures of the wires is shown in figure 4. If the damping properties are maintained at the maximum service temperatures, 3D woven metallic lattice materials would offer the damping properties of polymers at temperatures in which only high temperature metallic and technical ceramics are applicable.

It is worth noting that $\mathrm{NiCr}$ wires also exhibit excellent high temperature corrosion resistance due to the formation of a $\mathrm{Cr}_{2} \mathrm{O}_{3}$ layer that passivates the surface [39-41]. Guilemany et al. [42] have reported the ability to tailor the sliding wear properties of $\mathrm{Cr}_{3} \mathrm{C}_{2}-\mathrm{NiCr}$ coatings, which opens the door to improvements in the damping characteristics through further processing. Moreover, the damping simulations point to the influence that architectural variability has on the interplay between frictional and inertial damping. Optimization of the underlying microarchitecture offers the opportunity to design $3 \mathrm{D}$ woven metallic lattice materials with increased and tunable damping properties. Combining mechanical damping with active cooling, flow regulation, and electrical conductivity would enable multi-functional materials for use in elevated temperature environments.

This study was funded by Defense Advanced Research Projects Agency (DARPA), in the Materials with Controlled Microstructural Architecture (MCMA) program, under award number W91CRB1010004 (Dr. Judah Goldwasser, program manager). The authors would like to thank Dr. Yong Zhang for elastic modulus measurements and Dr. Harold Kahn for dynamic friction 
measurements. The simulations used the Extreme Science and Engineering Discovery Environment (XSEDE) that is supported by National Science Foundation grant number OCI1053575.

\section{References}

[1] M. Ashby, Metal Foams : A Design Guide, Butterworth-Heinemann, Boston, 2000.

[2] L.J. Gibson, M.F. Ashby, M. Ashby, Cellular Solids: Structure and Properties, 2nd ed., Cambridge University Press, 1999.

[3] J. Zhou, M.Z. Hassan, Z. Guan, W.J. Cantwell, Compos. Sci. Technol. 72 (2012) 1781.

[4] S.T. Szyniszewski, B.H. Smith, J.F. Hajjar, B.W. Schafer, S.R. Arwade, Mater. Des. 54 (2014) 1083.

[5] S. Szyniszewski, B.H. Smith, J.F. Hajjar, S.R. Arwade, B.W. Schafer, Thin-Walled Struct. 59 (2012) 11.

[6] M. Moradi, S.R. Arwade, B.W. Schafer, Thin-Walled Struct. 62 (2013) 206.

[7] J. Banhart, H.-W. Seeliger, Adv. Eng. Mater. 10 (2008) 793.

[8] A.H. Brothers, R. Scheunemann, J.D. DeFouw, D.C. Dunand, Scr. Mater. 52 (2005) 335.

[9] T.A. Schaedler, A.J. Jacobsen, A. Torrents, A.E. Sorensen, J. Lian, J.R. Greer, L. Valdevit, W.B. Carter, Science 334 (2011) 962.

[10] K.J. Maloney, K.D. Fink, T.A. Schaedler, J.A. Kolodziejska, A.J. Jacobsen, C.S. Roper, Int. J. Heat Mass Transf. 55 (2012) 2486.

[11] D.J. Sypeck, H.N.G. Wadley, J. Mater. Res. 16 (2001) 890.

[12] H.N.G. Wadley, K.P. Dharmasena, M.Y. He, R.M. McMeeking, A.G. Evans, T. BuiThanh, R. Radovitzky, Int. J. Impact Eng. 37 (2010) 317.

[13] K.P. Dharmasena, D.T. Queheillalt, H.N.G. Wadley, P. Dudt, Y. Chen, D. Knight, A.G. Evans, V.S. Deshpande, Eur. J. Mech. - ASolids 29 (2010) 56.

[14] C.J. Yungwirth, H.N.G. Wadley, J.H. O'Connor, A.J. Zakraysek, V.S. Deshpande, Int. J. Impact Eng. 35 (2008) 920.

[15] V.J. Challis, J.K. Guest, J.F. Grotowski, A.P. Roberts, Int. J. Solids Struct. 49 (2012) 3397.

[16] L. Zhao, S. Ha, K.W. Sharp, A.B. Geltmacher, R.W. Fonda, A.H. Kinsey, Y. Zhang, S.M. Ryan, D. Erdeniz, D.C. Dunand, K.J. Hemker, J.K. Guest, T.P. Weihs, Acta Mater. 81 (2014) 326. 
[17] Yong Zhang, Seunghyun Ha, Keith Sharp, James K. Guest, Tim Weihs, Kevin J. Hemker, Mater. Des. (n.d.).

[18] C. Lalanne, C. Lalanne, Fatigue Damage, 1st ed., CRC Press, 2002.

[19] J.W. Tedesco, W.G. McDougal, C.A. Ross, Structural Dynamics: Theory and Applications, Addison Wesley Longman, Menlo Park, Calif., 1999.

[20] S. Szyniszewski, T. Krauthammer, Eng. Struct. 42 (2012) 142.

[21] S. Szyniszewski, in:, 2009 Struct. Congr. - Dont Mess Struct. Eng. Expand. Our Role April 302009 - May 2 2009, American Society of Civil Engineers, Austin, TX, United states, 2009, pp. 1259-1268.

[22] D.W. Childs, J. Eng. Gas Turbines Power 100 (1978) 48.

[23] B.H. Ertas, H. Luo, J. Eng. Gas Turbines Power 130 (2008) 032503.

[24] B. Ertas, H. Luo, D. Hallman, Dynamic Characteristics of Shape Memory Alloy Metal Mesh Dampers, in: American Institute of Aeronautics and Astronautics. 50th AIAA/ASME/ASCE/AHS/ASC Structures, Structural Dynamics, and Materials Conference. 4 - 7 May 2009, Palm Springs, California.

[25] A. Bogdanovich, in:, Proceeding 16th Int. Conf. Compos. Mater., 2007.

[26] Keith Sharp, D. Mungalov, J. Brown, in:, Procedia Mater. Sci. 4, 2014, pp. 15-20.

[27] J.K. Guest, J.H. Prévost, Int. J. Solids Struct. 43 (2006) 7028.

[28] B.J. Lazan, Damping of Materials and Members in Structural Mechanics, Pergamon Press, 1968.

[29] E.J. Graesser, C.R. Wong, Mech. Mech. Mater. Damping Rep. No DTRC-SME-9105 David Taylor Res. Cent. Annap. MD (1992) 316.

[30] W. Liu, Experimental and Analytical Estimation of Damping in Beams and Plates with Damping Treatments, University of Kansas, 2008.

[31] M.F. Ashby, Materials Selection in Mechanical Design, Butterworth-Heinemann, Burlington, MA, 2011.

[32] M. Carfagni, E. Lenzi, M. Pierini, in:, n.d.

[33] Granta Designs Ltd., The CES EduPack '14, Granta Design Ltd., Cambridge, UK, 2014.

[34] J. Hallquist, LS-DYNA Theory Manual, Lawrence Software Technology Corporation, Livermore, California, 2006.

[35] J.-H. Ouyang, X.-S. Liang, Z.-G. Liu, Z.-L. Yang, Y.-J. Wang, Wear 301 (2013) 820.

[36] Hal Kahn (Private Communications), (2014).

[37] E.A. Avallone, T. Baumeister, L.S. Marks, Marks' Standard Handbook for Mechanical Engineers, 10th Revised edition, McGraw-Hill Publishing Co., 1996.

[38] Arcor Electronics, 2014.

[39] D.L. Douglass, Corros. Sci. 8 (1968) 665.

[40] F.H. Stott, G.C. Wood, Corros. Sci. 11 (1971) 799.

[41] W.F. Chu, A. Rahmel, Metall. Trans. B 10 (1979) 401.

[42] J.. Guilemany, J.. Miguel, S. Vizcaíno, C. Lorenzana, J. Delgado, J. Sánchez, Surf. Coat. Technol. 157 (2002) 207.

[43] David I. Jones, Results of a Round Robin Test Program: Complex Modulus Properties of a Polymeric Damping Material (Wright Lab, Wright-Patterson AFB OH, May 1992), ADA263619. 
Table 1. Sensitivity of the simulated loss coefficient to wire spacing and the coefficient of friction in the 3D woven modified architecture lattice of $\mathrm{NiCr}$.

\begin{tabular}{|l|c|c|c|}
\hline Gaps & No gaps & $\begin{array}{c}\text { Horizontal } \\
\text { gaps only }\end{array}$ & $\begin{array}{c}\text { Average } \\
\text { measured } \\
\text { gaps }\end{array}$ \\
\hline Simulated gap spacings (microns) & $\begin{array}{c}\text { warp }=0 \\
\text { fill }=0 \\
\mathrm{z}=0\end{array}$ & $\begin{array}{c}\text { warp }=99 \\
\text { fill }=10 \\
\mathrm{z}=0\end{array}$ & $\begin{array}{c}\text { warp }=99 \\
\text { fill }=10 \\
\mathrm{z}=47\end{array}$ \\
\hline Measured coefficient of friction $\left(f_{s}=0.44, f_{d}=0.20\right)$ & $\mathbf{0 . 1 5}$ & $\mathbf{0 . 1 3}$ & $\mathbf{0 . 0 6}$ \\
\hline Increased coefficient of friction $\left(f_{s}=0.88, f_{d}=0.40\right)$ & $\mathbf{0 . 1 9}$ & $\mathbf{0 . 1 4}$ & $\mathbf{0 . 0 6}$ \\
\hline Comments & $\begin{array}{c}\text { Sensitive } \\
\text { to friction }\end{array}$ & $\begin{array}{c}\text { Minor } \\
\text { friction } \\
\text { effect }\end{array}$ & $\begin{array}{c}\text { Negligible } \\
\text { friction } \\
\text { effect }\end{array}$ \\
\hline
\end{tabular}


Figures:
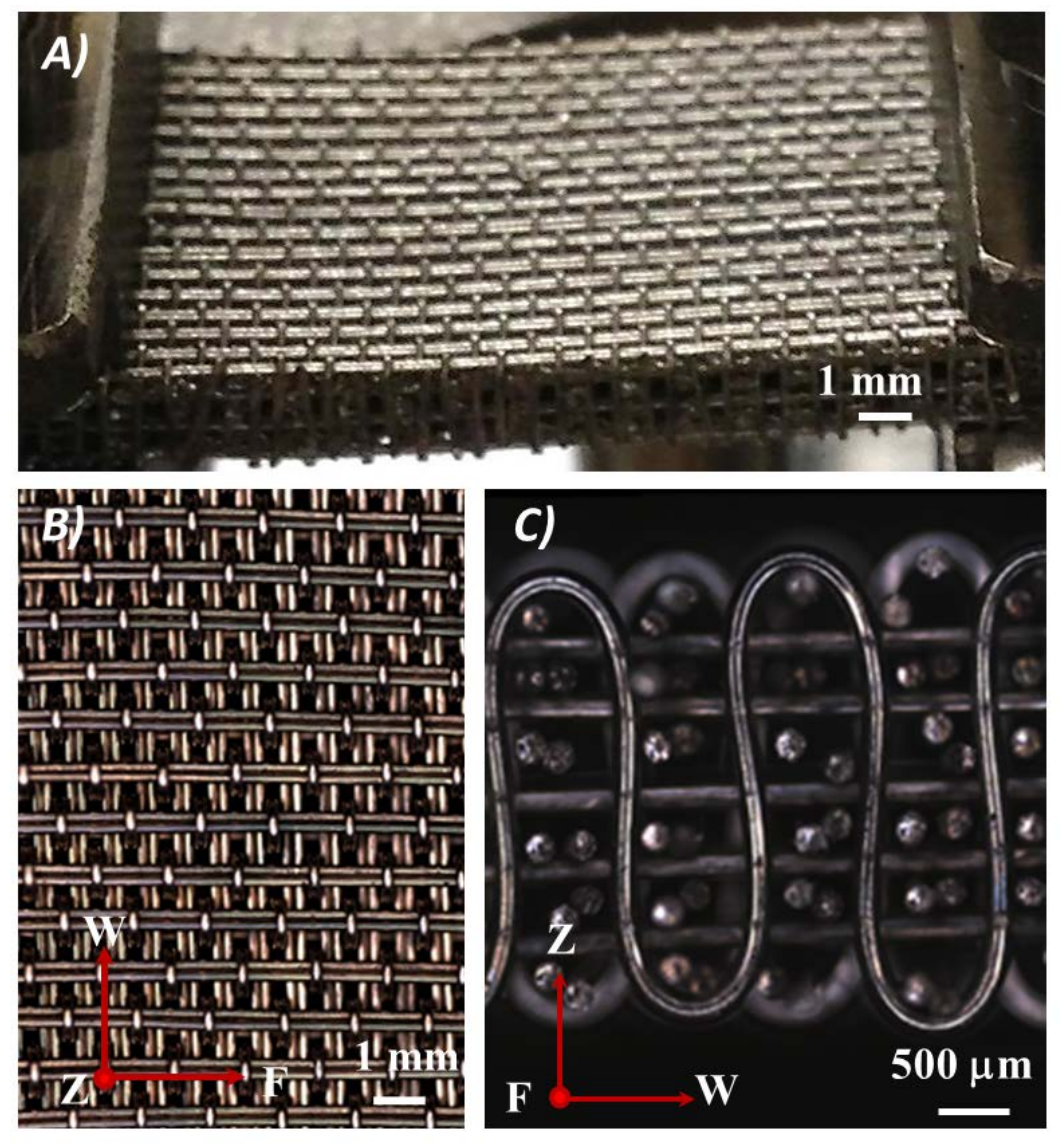

Figure 1. A) Metallic 3D woven sample clamped in the DMA, B) top view of the lattice material, C) side view of the standard material exhibiting gaps between many of the wires. $\mathrm{W}, \mathrm{F}$, and $\mathrm{Z}$ represent the warp, fill, and $\mathrm{Z}$ directions respectively. 


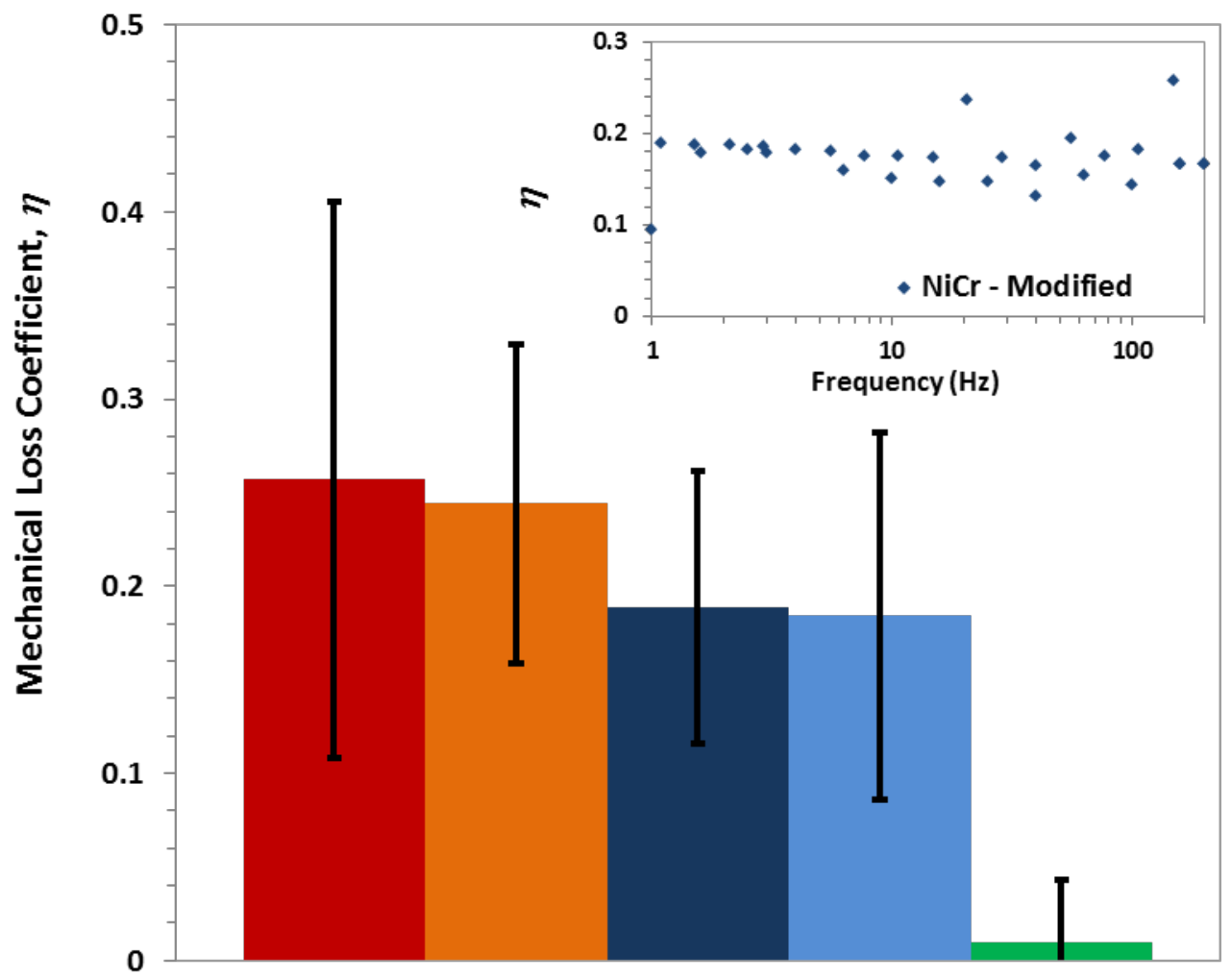

$\square$ Cu-modified $\square$ Cu-standard $\square$ NiCr-modified $\square$ NiCr-standard $\square$ Cu-solid plate

Figure 2. Averaged experimental loss coefficients, $\eta$ (damping) of the 3D woven metallic lattice materials. The inset illustrates that the loss factor for the NiCr-modified architectured material did not depend on frequency and is representative of all of the samples. 


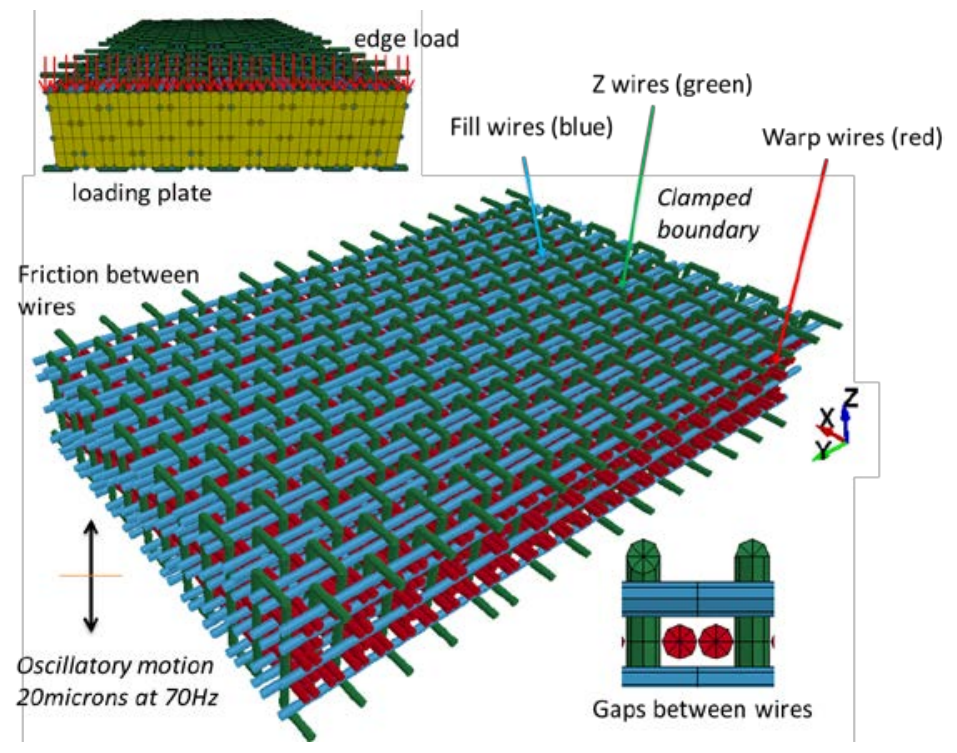

Figure 3. Simulation of the 3D woven metallic sample (clamped at far end, and subjected to oscillatory excitation at the near end). The model employs frictional contact. The modified weave architecture is shown with fill wires shaded blue, the warp wires shaded red, and the z-wires shaded green. 


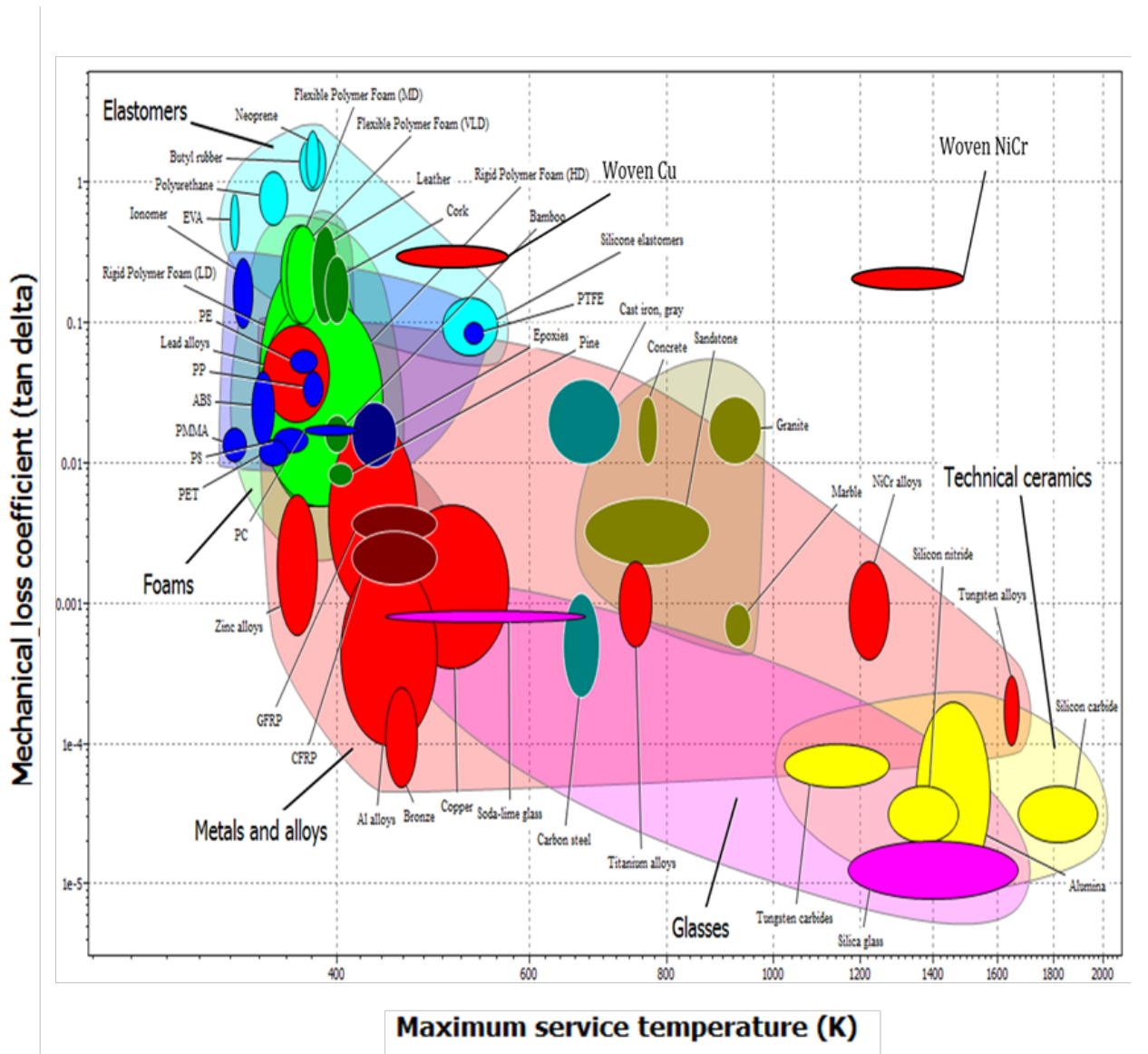

Figure 4. A property correlation plot of the mechanical loss factor, $\eta$, and the maximum service temperature for a wide variety of materials and material classes. The measured damping performance of 3D woven $\mathrm{NiCr}$ lattice materials combined with the maximum service temperature of $\mathrm{NiCr}$ highlight its potential for use in elevated temperature damping environments. Note that the woven materials were all measured in bending. The reference values may have been obtained with different loading conditions, but Jones et al have reported similar values for loss factors obtained in tension and bending [43]. 